\title{
On the importance of the influence of both velocity and aspect ratios on the occurrence of bifurcation phenomena within a two-sided lid-driven enclosure
}

\author{
Fayçal HAMMAMI, Nader BEN-CHEIKH and Brahim BEN-BEYA \\ Laboratory of Fluid Dynamics, Physics Department, Faculty of Science of Tunis, Campus \\ Universitaire, 2092 El-Manar II, Tunisia.
}

Keywords: Two-sided lid-driven cavity, critical Reynolds number, Hopf bifurcation, shallow aspect ratios, deep cavity flow.

\begin{abstract}
This paper deals with the numerical study of bifurcations in a two-sided lid driven cavity flow. The flow is generated by moving the upper wall to the right while moving the left wall downwards. Numerical simulations are performed by solving the unsteady two dimensional NavierStokes equations using the finite volume method and multigrid acceleration. In this problem, the ratio of the height to the width of the cavity are ranged from $H / L=0.25$ to 1.5 . The code for this cavity is presented using rectangular cavity with the grids $144 \times 36,144 \times 72,144 \times 104,144 \times$ $136,144 \times 176$ and $144 \times 216$. Numerous comparisons with the results available in the literature are given. Very good agreements are found between current numerical results and published numerical results.

Various velocity ratios ranged in $0.01 \leq \alpha \leq 0.99$ at a fixed aspect ratios $(\mathrm{A}=0.5,0.75,1.25$ and 1.5$)$ were considered.

It is observed that the transition to the unsteady regime follows the classical scheme of a Hopf bifurcation. The stability analysis depending on the aspect ratio, velocity ratios $\alpha$ and the Reynolds number when transition phenomenon occurs is considered in this paper.
\end{abstract}

\section{Nomenclature}

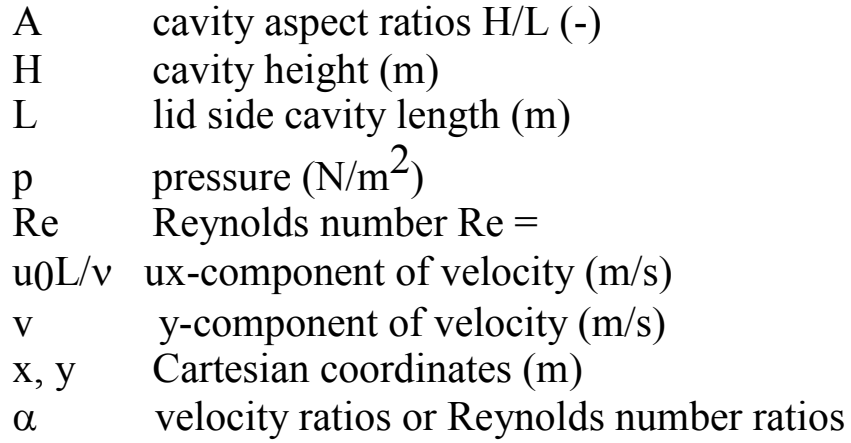

\section{INTRODUCTION}

Lid-driven cavity (LDC) flows have been a mainstay of fluid dynamics research for decades [1]. By far, the most studied configurations are that of two-dimensional flow set up by constant speed motion of a flat surface over a square or rectangular container. For instance, one can refer to the first numerical solutions of the LDC problem with Burggraf [2].

A classic example is the case where a flow is induced by the tangential movement of either one or both facing cavity boundaries (i.e. one-sided lid-driven cavity flow or two-sided lid-driven cavity flow, respectively). For example, Fortin et al. [3], Erturk et al [4] and Ding et al.[5] focused on numerical methods in order to test the One-sided lid-driven flow in a square cavity

A numerical and an experimental investigations have been carried out by Kuhlmann et al. [6,7] to extend the one-sided lid-driven cavity flow problem in the two- and three dimensional flows, 
where the two moving lids face each other and experience either parallel or anti-parallel motion. At a low Reynolds number, the flow consists of separate co-rotating vortices adjacent to each of the moving walls. At high Reynolds numbers, the cat's eye flow becomes unstable and transforms into a steady three-dimensional cellular flow.

Patil et al. [8] numerically studied the lid driven cavity flow for the aspect ratios of 1 to 4 in different Reynolds number ranging from 50 until 3200. An experimental investigations have been carried out by Taneda [9] to study the effect of the aspect ratio on the laminar regime.

Wahba [10] numerically studied the two-sided lid driven cavity flows at low Reynolds number. It was observed that the flow generally remains steady then an instability occurs when the Reynolds number increases.

A recent work has been carried out by [11], who applied the lattice Bhatnagar-Gross- Krook (LBGK) model to compute two-adjacent-lid-driven cavity flows.

In another contribution, Perumal and Dass [12], numerically simulated the two-sided lid-driven square cavity flow driven by parallel and anti-parallel motion at different Reynolds numbers using finite difference method and Lattice Boltzmann method.

By increasing the Reynolds number, the accurate solution of the equations becomes more difficult (see Fortin et al. [13], Peng et al. [14] etc). More recently, Bopanna and Gajjar [15] introduced a number of techniques and algorithms to obtain an accurate solution of the equation for high Reynolds numbers.

The focus of this paper is to estimate the critical Reynolds number in a 2D flow for various aspects (depth/width), Reynolds number and velocity ratios $\alpha$.

In the present study we have used a robust and an accurate numerical technique to first obtain the steady flow. The same methodology is used to investigate the stability of the flow by solving the generalized two-dimensional eigen value problem obtained from the linearized unsteady equations. The details of the numerical techniques used are discussed in Section 2 . Then in Section 3, we present various results on the regularized driven cavity flow for a wide range of the Reynolds number.

\section{NUMERICAL DETAILS}

\subsection{Governing equations}

Lid-driven cavity flows have been a perennial topic of research in the fluid mechanics community for years. The prevalent assumptions for lid-driven cavity flows revolving around two-dimensional flow, incompressible and viscous fluid seek to simplify the Navier-Stokes equations drastically. The boundary conditions to be imposed are dictated by the type of velocity at the wall, either stationary or moving.

Fluid motion inside the lid-driven cavity is governed by the unsteady Navier-Stokes equations written in tensor notation:

$\left\{\begin{array}{l}\frac{\partial u_{i}}{\partial x_{i}}=0 \\ \frac{\partial u_{i}}{\partial t}+\frac{\partial\left(u_{i} u_{j}\right)}{\partial x_{j}}=-\frac{\partial p}{\partial x_{i}}+\frac{1}{\operatorname{Re}} \frac{\partial^{2} u_{i}}{\partial x_{j} \partial x_{j}}\end{array}\right.$

Here, the set of dimensionless quantities $\mathrm{x}_{\mathrm{i}}(\mathrm{x}, \mathrm{y}), \mathrm{u}_{\mathrm{i}}(\mathrm{u}, \mathrm{v}), \mathrm{p}$ and $\mathrm{t}$ denotes the coordinate space, the velocity component in each $x_{\mathrm{i}}$ direction, the hydrodynamic pressure and time, respectively. Also, the body forces have been included in the pressure term. The configuration of the two-dimensional, of width $L$, height $H$ is sketched in Fig. 1. The cavity has a span-wise aspect ratio $A=H / L$ and filled with an incompressible flow of a Newtonian fluid with constant viscosity and constant density. 
Where $\operatorname{Re}=\frac{u_{0} H}{v}$ is the non-dimensional Reynolds number.

According to the geometric model presented in Fig. 1, the velocity boundary conditions can be written as follows :

- At $\mathrm{y}=\mathrm{A}: u_{0}=1, v_{0}=0$

- At $x=0: u_{0}=0, v_{0}=-\alpha u_{0} ;$ - At $x=1: u_{0}=v_{0}=0$;

- At $y=0: u_{0}=v_{0}=0$.

The velocity ratios or Reynolds number ratios is $\alpha=\frac{v_{0}}{u_{0}}=\frac{\operatorname{Re}_{\mathrm{v}}}{\operatorname{Re}_{\mathrm{u}}}$.

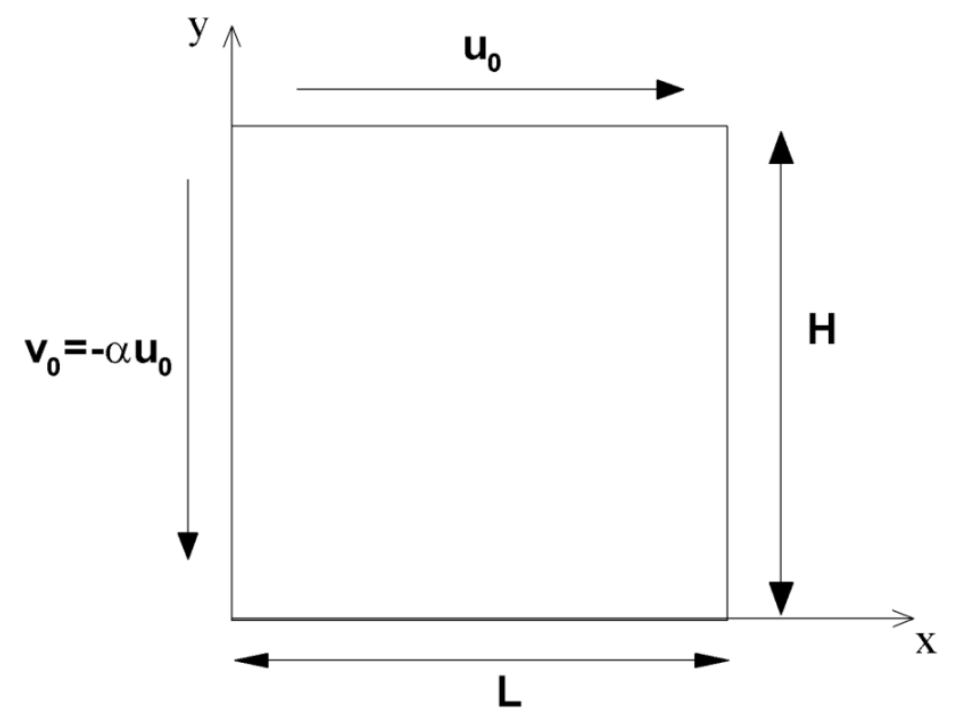

Fig. 1. Geometric description of the lid driven cavity flow.

\subsection{Numerical procedure}

Following Patankar [16] the unsteady Navier-Stokes equations are discretized using staggered, nonuniform control volumes. A projection method attributed to Achdou and Guermond [17] is employed to couple the momentum and continuity equations. An intermediate velocity is first computed and later updated to comply with mass continuity. In the intermediate velocity field, the old pressure is used. A Poisson equation having the divergence of the intermediate velocity field as the source term is solved. After this, the pressure correction is obtained first and the real velocity field second. Meanwhile, the time-dependent terms are discretized with an implicit second-order Euler scheme as follows:

$$
\left(\frac{\partial \phi}{\partial t}\right)^{n+1}=\frac{3 \phi^{n+1}-4 \phi^{n}+\phi^{n-1}}{2 \Delta t}
$$

where $\phi$ is a generic velocity that stands for $u$ or $v$. The advection terms are discretized with an explicit scheme, which is conveniently expressed by

$$
\frac{\partial}{\partial x_{j}}\left(u_{i} u_{j}\right)^{n+1}=2 \frac{\partial}{\partial x_{j}}\left(u_{i} u_{j}\right)^{n}-\frac{\partial}{\partial x_{j}}\left(u_{i} u_{j}\right)^{n-1}
$$

The finite-volume method[16] is adopted to discretize the Navier-Stokes equations. The advective terms in the momentum equation are discretized using a QUICK third-order scheme proposed by Leonard [18]. The discretized momentum equations are resolved employing the red and black successive over relaxation method RBSOR (Barrett et al.[19]), while the Poisson pressure correction equation is solved by way of a full multi-grid method as suggested by Ben-Cheikh et 
al.[20] The numerical method is implemented on a FORTRAN program. Convergence of the numerical results is verified at each time step according to the stringent criterion:

$$
\sqrt{\sum_{i, j} \phi_{i, j}^{m}-\sum_{i, j} \phi_{i, j}^{m-1}} \leq 10^{-6}
$$

\section{RESULTS AND DISCUSSION}

\subsection{Validation of the numerical method}

To validate the numerical simulation, the single sided lid-driven square cavity flow been compared with those obtained by Omari [21] with a CFD code for a fixed value $R e=1000$. It is worth noting that we used a grid size of $144 \times 72$ and $144 \times 216$ for both aspect ratios $\mathrm{A}=0.5$ and $\mathrm{A}=1.5$, respectively. Figures 2 (a) and 2(b) display the profiles of the u-velocity component along the vertical centerline. This comparison reveals excellent agreements between the present results and those obtained by Omari [21]. The close agreement gives credibility to the result of the present finite volume code and it stands validated.

(a)

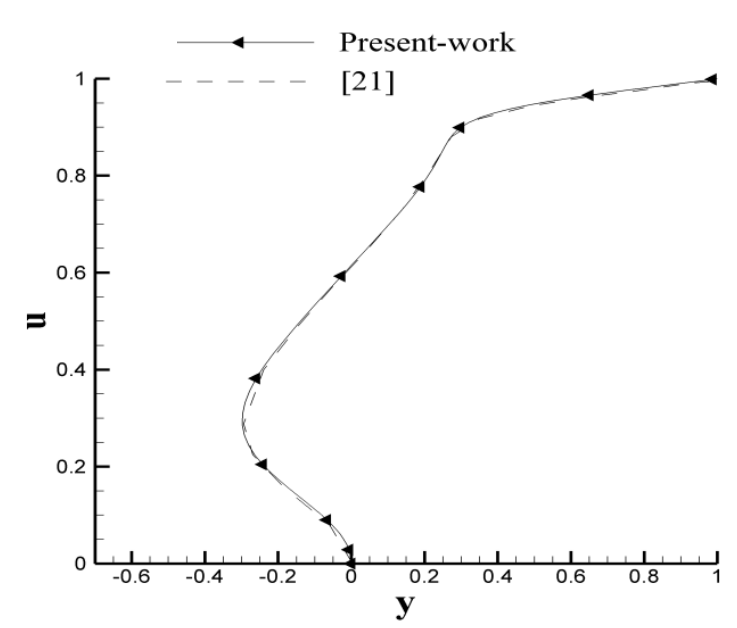

(b)

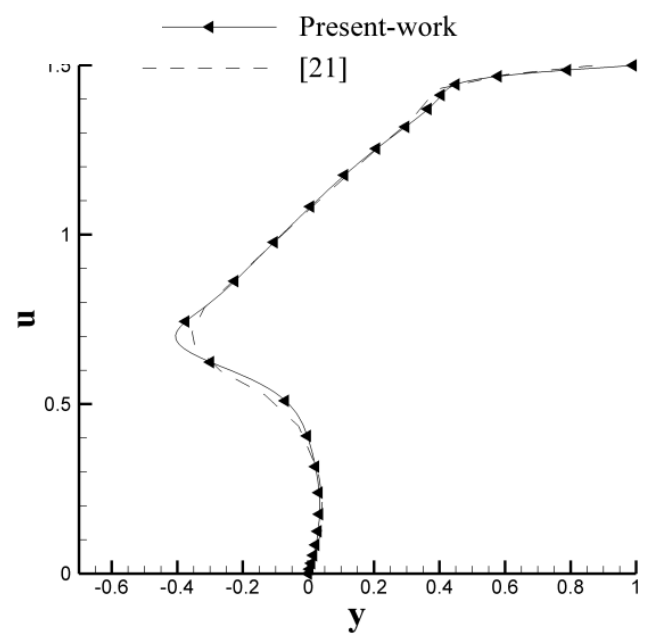

Fig. 2. Comparison of the $u$-velocity component distribution along the centerline of lid-driven square cavity of the present work with Omari. [21] for (a) $A=0.5$ and (b) $A=1.5$ at $\operatorname{Re}=1000$.

\subsection{Location of the $2 \mathrm{D}$ critical Reynolds numbers for different aspect ratio $\mathrm{A}$.}

The stability analysis of the two lid driven cavity flow is investigated using finite volume method for various aspect ratios.

To put the critical Reynolds number, we found that the steady flow became unstable via a critical Hopf bifurcation. The steady flow was replaced by a periodic flow when Re exceeds a threshold value. It is known, for the Hopf bifurcation, that the amplitude of the solution grows with the square-root of the bifurcation parameter. In our case, this means that the square of a velocity component should be proportional to the Reynolds number Re, the critical Reynolds number after the bifurcation has taken place. 

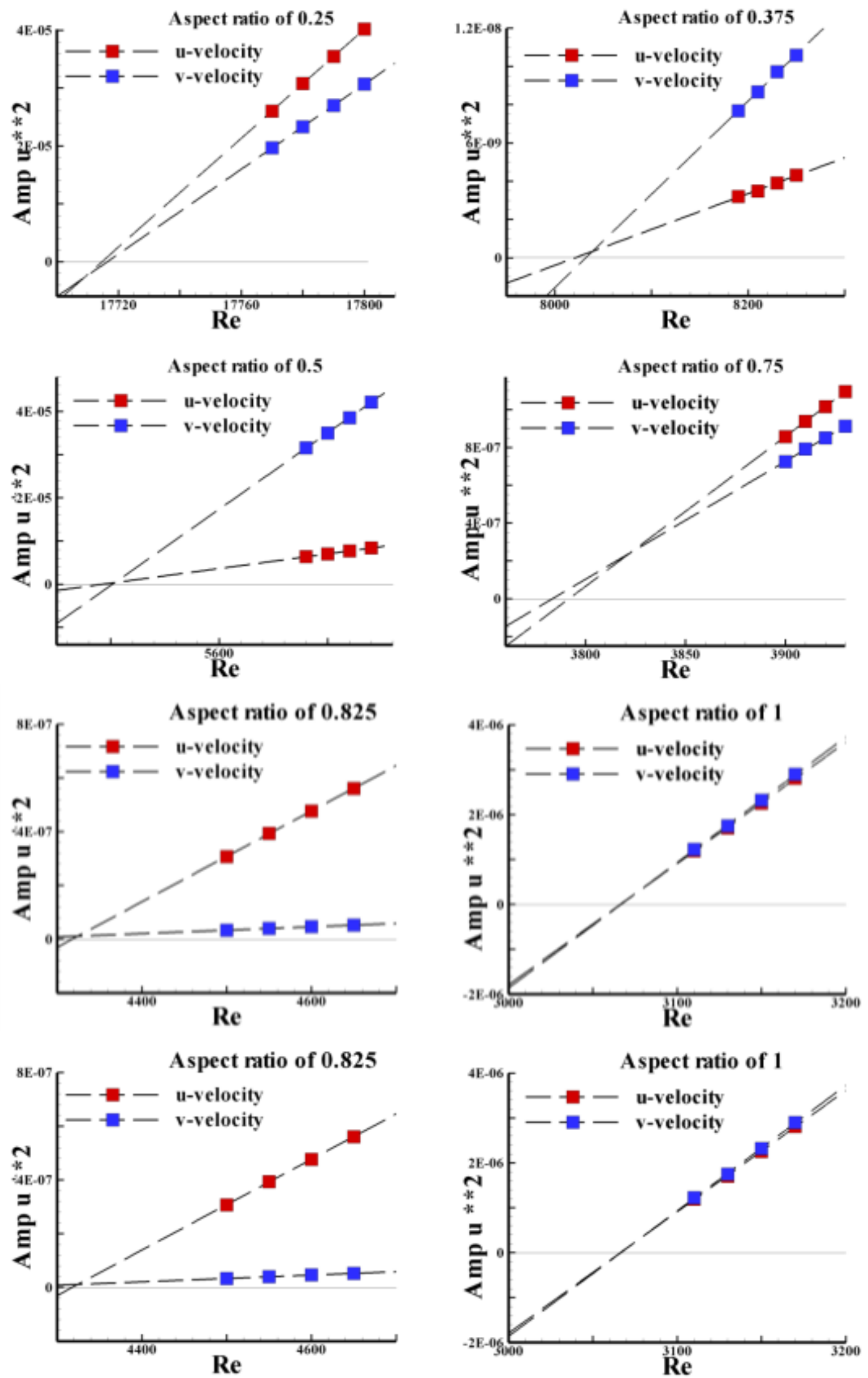

Fig. 3. Square of the u- and v-velocity components as a function of the cavity aspect ratios magnitude fluctuations against the Reynolds number. 
As illustrated in Fig. 3. The plots of the square amplitude of the fluctuation of $u$ - and v-velocity components versus the Reynolds number are approximately straight lines. These lines can be extrapolated to where it crosse the zero axis to approximate the bifurcation point which gives a critical value of the Reynolds number $R e$, associated with various aspect ratios $A=0.25, A=0.375$, $\mathrm{A}=0.5,0.75, \mathrm{~A}=0.825$ and $\mathrm{A}=1$, respectively. Besides, the neutral curve (critical Re versus $\mathrm{A}$ ) is reported in Fig. 4 for the two-sided lid-driven cavity problem. As illustrated in this figure, Rec decreases as $\mathrm{A}$ was augmented from $\mathrm{A}=0.25$ to $\mathrm{A}=0.75$, then increases from $\mathrm{A}=0.75$ to $\mathrm{A}=$ 0.825 to reach its minimum value $(\operatorname{Rec}=3066.51)$ at $\mathrm{A}=1$.

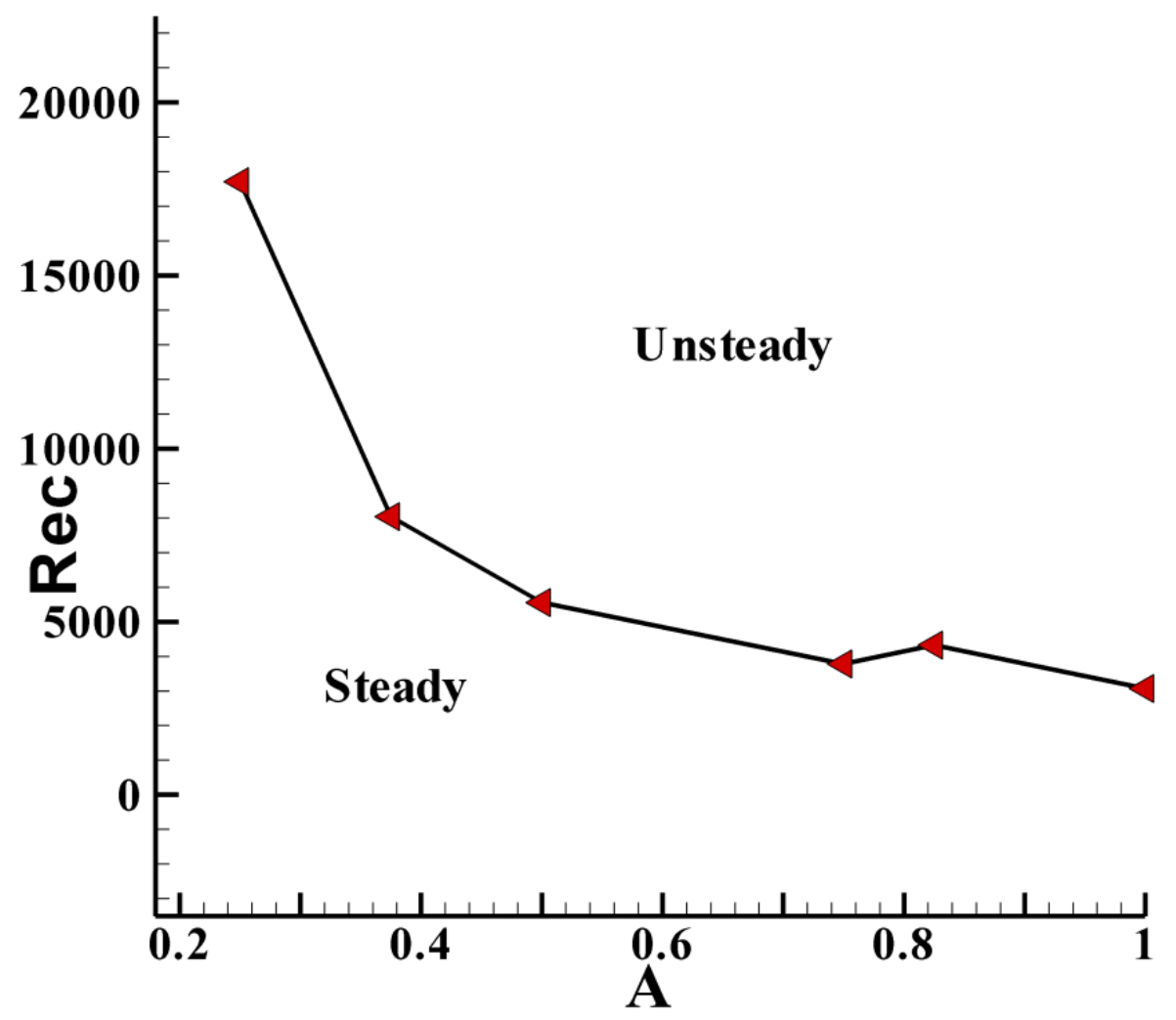

Fig. 4. Critical Reynolds number versus different aspect ratios A.

The values of the critical Reynolds numbers Rec are reported in Table 1. Note that Rec is determined by taking the mean of both values that surface up from the extrapolation of $\mathrm{u}$ and $\mathrm{v}$ amplitudes, respectively.

Table 1 : Numerical values of the critical Reynolds number where the first Hopf-bifurcation occurs.

\begin{tabular}{lcccccc}
\hline Aspect ratios & 0.25 & 0.375 & 0.5 & 0.75 & 0.825 & 1 \\
\hline Rec & 17691.10 & 8026.63 & 5548.53 & 3787.63 & 4276.88 & 3066.51
\end{tabular}

\subsection{Location of the $2 \mathrm{D}$ critical Reynolds numbers for different velocity ratio}

The same approach used for the 2D square cavity flow $(A=1)$ is extended to localize the Hop bifurcation in the two-sided lid-driven cavity flow at various velocity ratios $\alpha$ for both adopted cases $\mathrm{A}<1$ and $\mathrm{A}>1$.

\subsubsection{Effects of Reynolds numbers and velocity ratio on bifurcation occurrence for $A<1$.}

In this section, we attempt to study the effect of velocity ratio $\alpha$ on the values of the critical Reynolds numbers were the Hopf bifurcation is reached for the shallow aspect ratios $\mathrm{A}<1$. Figure 5 
reflects the square amplitude $\mathrm{Amp}^{2}$ as a function of Re associated with various aspect ratios $\mathrm{A}=0.5$ and $\mathrm{A}=0.75$. It is to mention that for each aspect ratio we have used the corresponding velocity ratio $\alpha=0.5$ and $\alpha=0.75$, respectively.

The values of the critical Reynolds numbers Rec for each aspect ratio and their corresponding velocity ratio $\alpha$ ranging in $0.01 \leq \alpha \leq 0.99$, are reported in Table 2 and Table 3 .

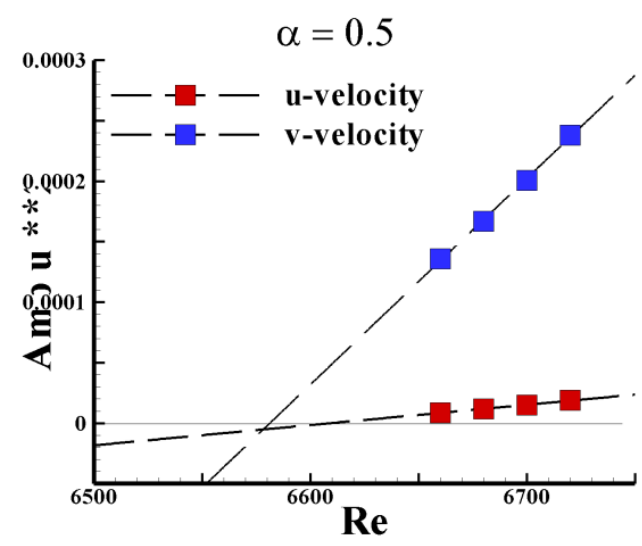

(a)
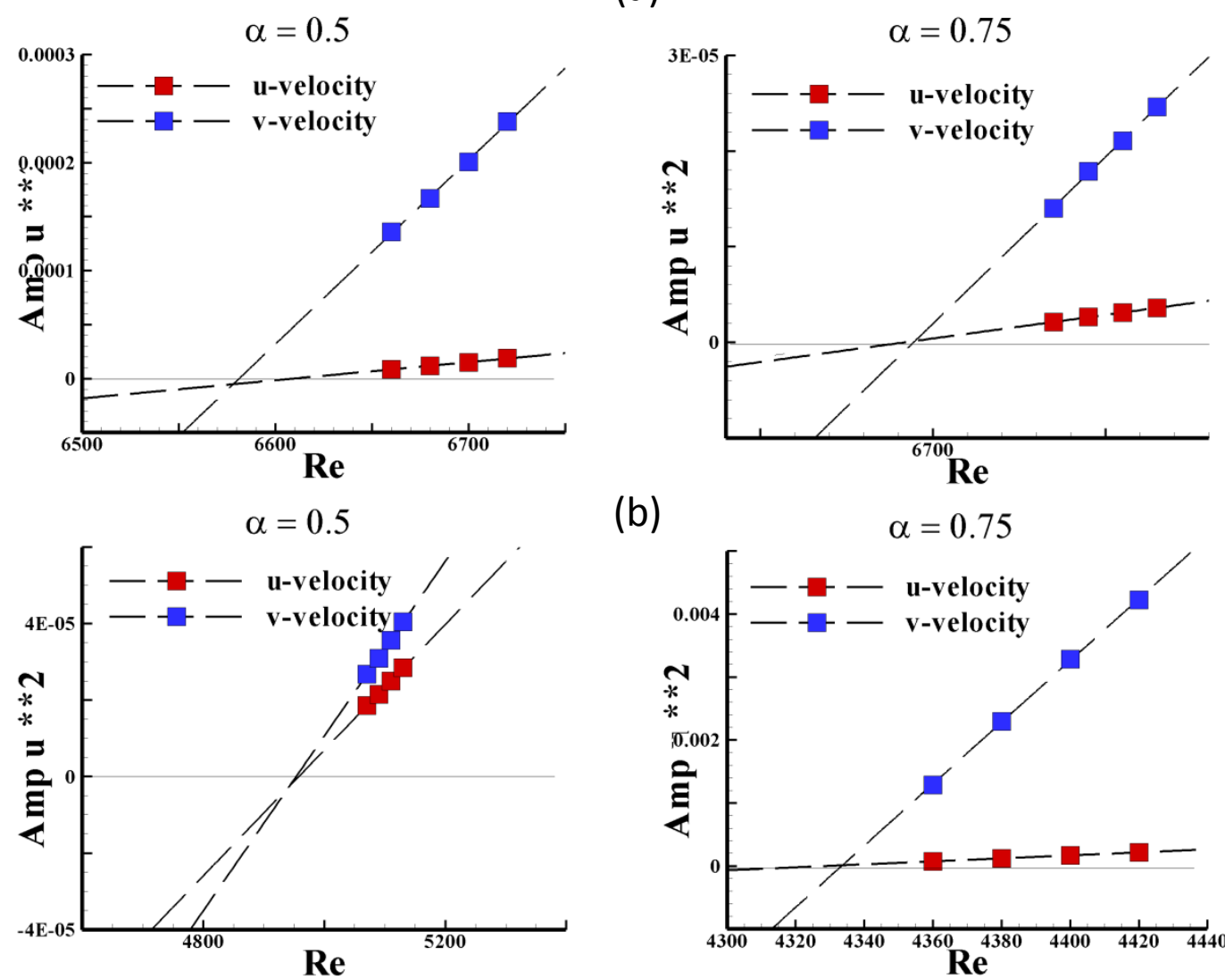

(b)

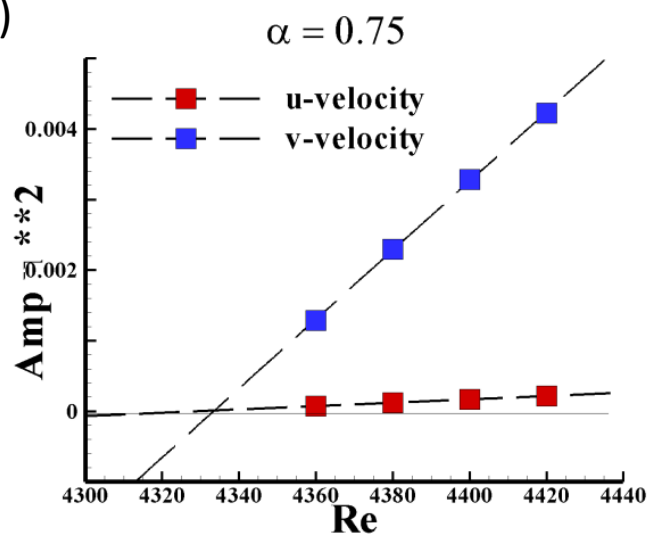

Fig. 5. Square of u- and v-velocity component magnitude fluctuations against the Reynolds number as a function of the velocity ratios for (a) $\mathrm{A}=0.5$ and (b) $\mathrm{A}=0.75$

Tab 2 : Computed values of the critical Reynolds number Re at different aspect ratio and corresponding velocity ratios $\alpha$ where the first Hopf-bifurcation occurs for $\mathrm{A}=0.5$.

\begin{tabular}{lllllll}
\hline & $\alpha=0.01$ & $\alpha=0.25$ & $\alpha=0.35$ & $\alpha=0.5$ & $\alpha=0.75$ & $\alpha=0.99$ \\
\hline Rec & 6548.93 & 6569.78 & 6617.69 & 6595.18 & 6693.16 & 5673.08 \\
\hline
\end{tabular}

Tab 3 : Computed values of the critical Reynolds number Re at different aspect ratio and corresponding velocity ratios $\alpha$ where the first Hopf-bifurcation occurs for $\mathrm{A}=0.75$.

\begin{tabular}{lllllll}
\hline & $\alpha=0.01$ & $\alpha=0.25$ & $\alpha=0.35$ & $\alpha=0.5$ & $\alpha=0.75$ & $\alpha=0.99$ \\
\hline Rec & 4879.88 & 1583.21 & 1631.42 & 4955.79 & 4330.97 & 3776.76 \\
\hline
\end{tabular}

In Fig. 6 is reported the variation of critical Reynolds number Rec with respect to $\alpha$ for $A=0.5$. As illustrated in this figure, Rec increases as $\alpha$ is augmented from $\alpha=0.01$ to $\alpha=0.825$, then decreases to reach its minimum value $(\operatorname{Rec}=5673.08)$ at $\alpha=0.99$. 
On another hand the variation of the critical Reynolds number Rec for $\mathrm{A}=0.75$ versus velocity ratio $\alpha$ ranging in $0.01 \leq \alpha \leq 0.99$ is plotted in Fig. 7. As illustrated in this figure, Rec decreases as $\alpha$ increased from 0.01 to 0.35 to reach its minimum value $(\operatorname{Rec} \approx 1011.07)$ at $\alpha \approx 0.3$. A peak is obtained at nearly $\alpha \approx 0.56$ after which the curve undergoes a decreasing trend.

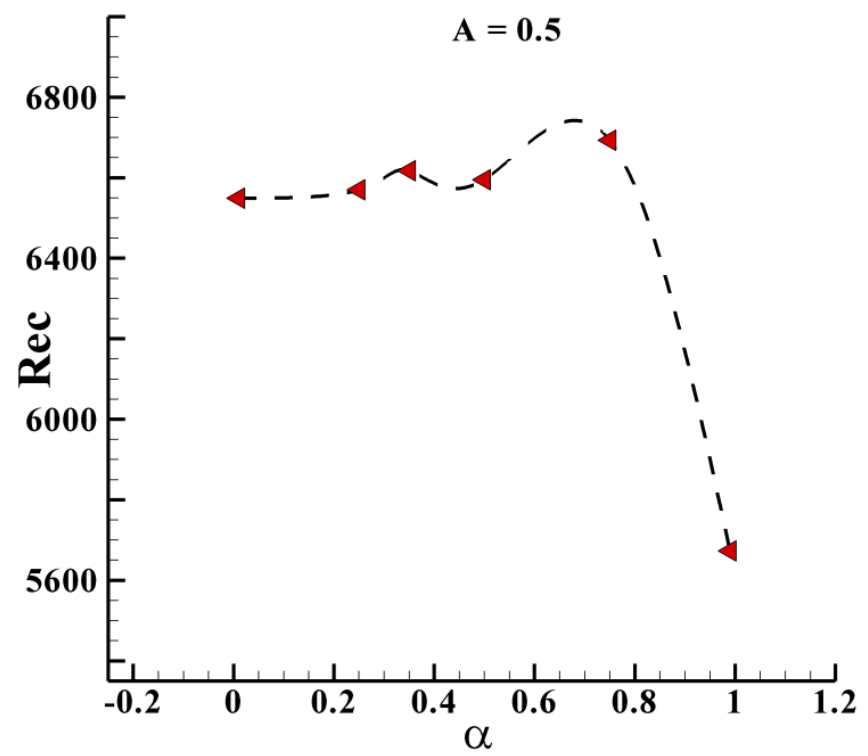

Fig. 6. Critical Reynolds number versus the velocity ratio $\alpha$, at $\mathrm{A}=0.5$.

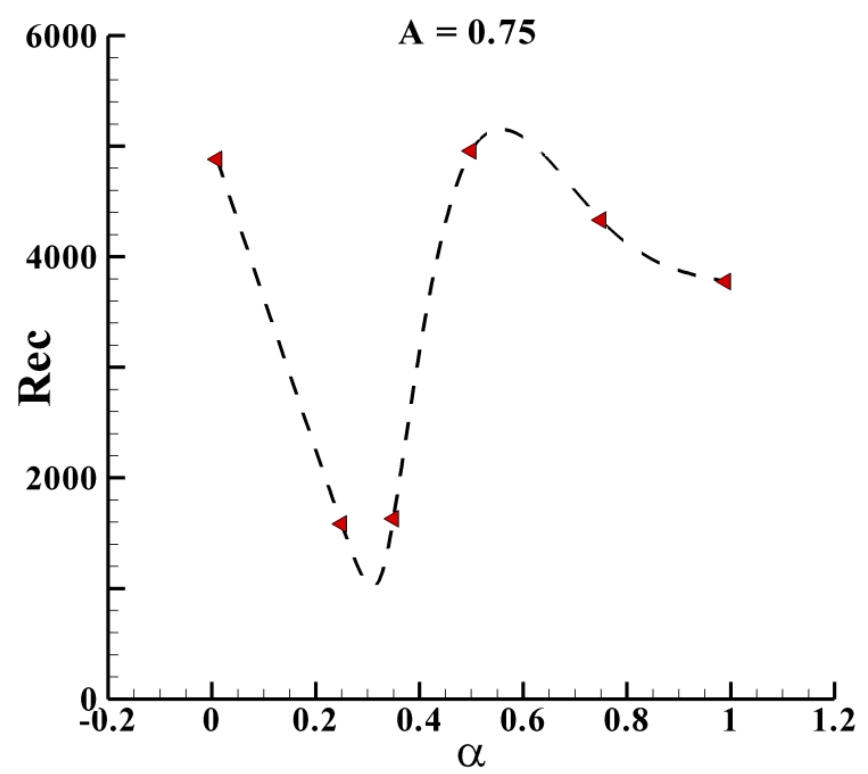

Fig. 7. Critical Reynolds number with respect to the velocity ratio $\alpha$, at $A=0.75$.

\subsubsection{Effects of Reynolds numbers and velocity ratio on the bifurcation appearance for $A>1$.}

In this section we focused on the effect of the deep aspect ratios $A>1$ and velocity ratio $\alpha$ on the values of the critical Reynolds numbers. Figures $(8 \mathrm{a}, 8 \mathrm{~b})$ displays the variation of $\mathrm{Amp}^{2}$ as a function of $\mathrm{Re}$ associated with various aspect ratios $\mathrm{A}=1.25, \mathrm{~A}=1.5$. For each aspect ratio we have used both values of the velocity ratios defined by $\alpha=0.01$ and $\alpha=0.99$, respectively. 
The values of the critical Reynolds numbers $\mathrm{Rec}$ for both aspect ratios $\mathrm{A}=1.25, \mathrm{~A}=1.5$ and their corresponding velocity ratio $\alpha$ ranging in $0.01 \leq \alpha \leq 0.99$, are reported in Table 4 and Table 5, respectively.

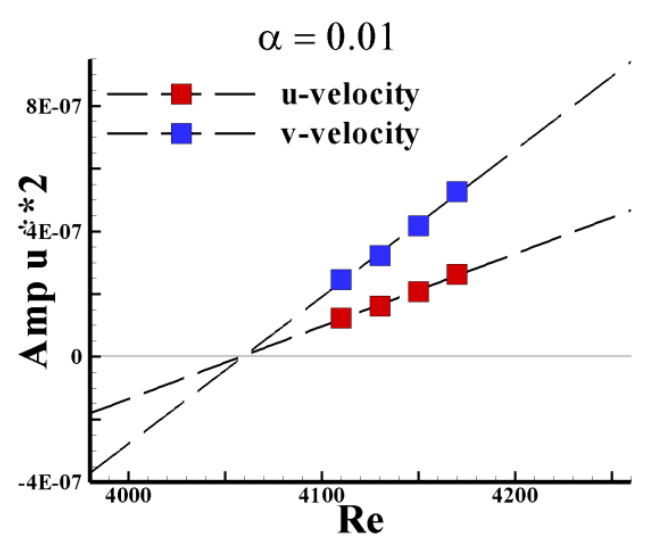

(a)

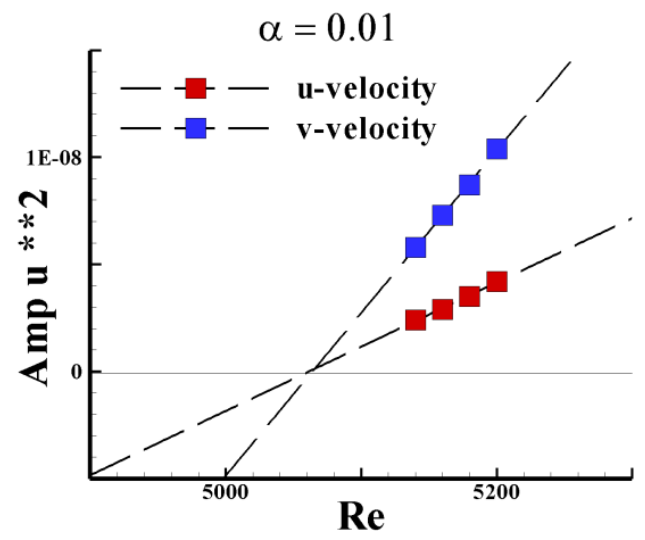

(b)
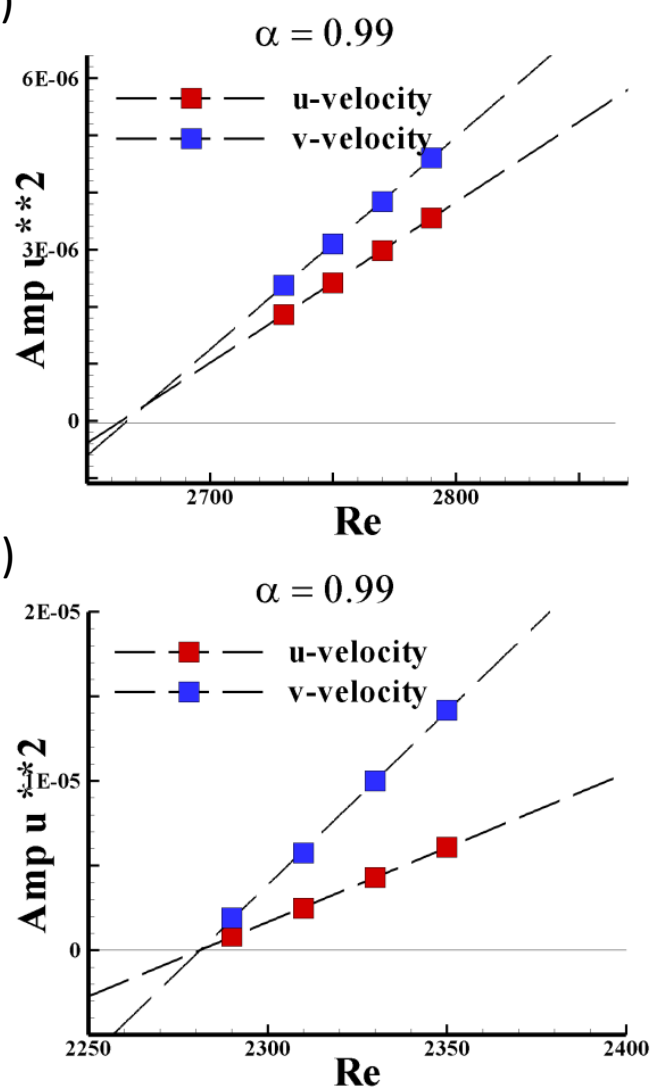

Fig. 8. Square of the $u$ and v-velocity component magnitude fluctuations against the Reynolds number as a function of the velocity ratios for (a) $\mathrm{A}=1.25$ and (b) $\mathrm{A}=1.5$.

Tab 4 : Computed values of the critical Reynolds number Re at different aspect ratio and corresponding velocity ratios where the first Hopf-bifurcation occurs for $\mathrm{A}=1.25$.

\begin{tabular}{lllllll}
\hline & $\alpha=0.01$ & $\alpha=0.25$ & $\alpha=0.35$ & $\alpha=0.5$ & $\alpha=0.75$ & $\alpha=0.99$ \\
\hline Rec & 4058.76 & 3721.25 & 3201.47 & 3337.37 & 3376.95 & 2665.17 \\
\hline
\end{tabular}

Tab 5 : Computed values of the critical Reynolds number Re at different aspect ratio and corresponding velocity ratios where the first Hopf-bifurcation occurs for $\mathrm{A}=1.5$.

\begin{tabular}{lllllll}
\hline & $\alpha=0.01$ & $\alpha=0.25$ & $\alpha=0.35$ & $\alpha=0.5$ & $\alpha=0.75$ & $\alpha=0.99$ \\
\hline $\operatorname{Rec}$ & 5060 & 4418.62 & 3454.23 & 3616.70 & 3476.20 & 2281.14
\end{tabular}

As illustrated in Fig. 9 and Fig.10, the critical Reynolds number Rec is reported for $A=1.25$. As illustrated in this figure, Rec decreases as $\alpha$ was increased from $\alpha=0.01$

to reach its minimum value $(\operatorname{Rec}=3201.47)$ at $\alpha=0.35$, then increases to reach its maximum value $(\operatorname{Rec} \approx 3434)$ at $\alpha \approx 0.6$ after which it continues to decrease. The same behavior was observed for an aspect ratio $\mathrm{A}=1.5$ with a minimun at $\alpha \approx 0.35$ and a maximum at $\alpha \approx 0.58$. The maximum value of $\mathrm{Rec}$ is found to be $(\operatorname{Rec} \approx 3680)$ for the velocity ratio ranging in $0.35 \leq \alpha \leq 0.99$. 


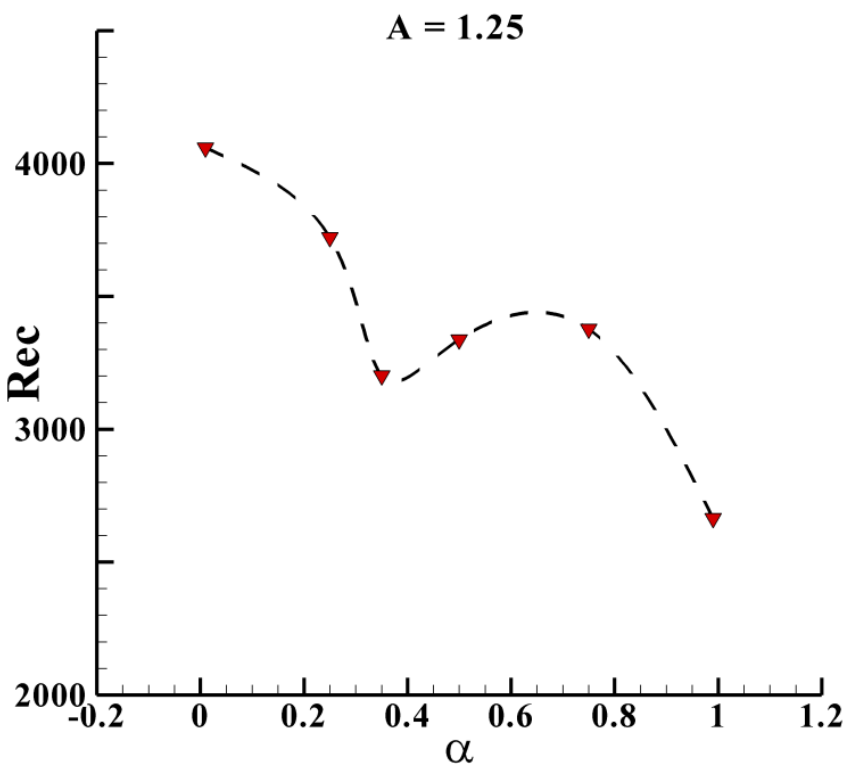

Fig. 9. Variation of the critical Reynolds number with respect to the velocity ratio $\alpha$, at $A=1.25$.

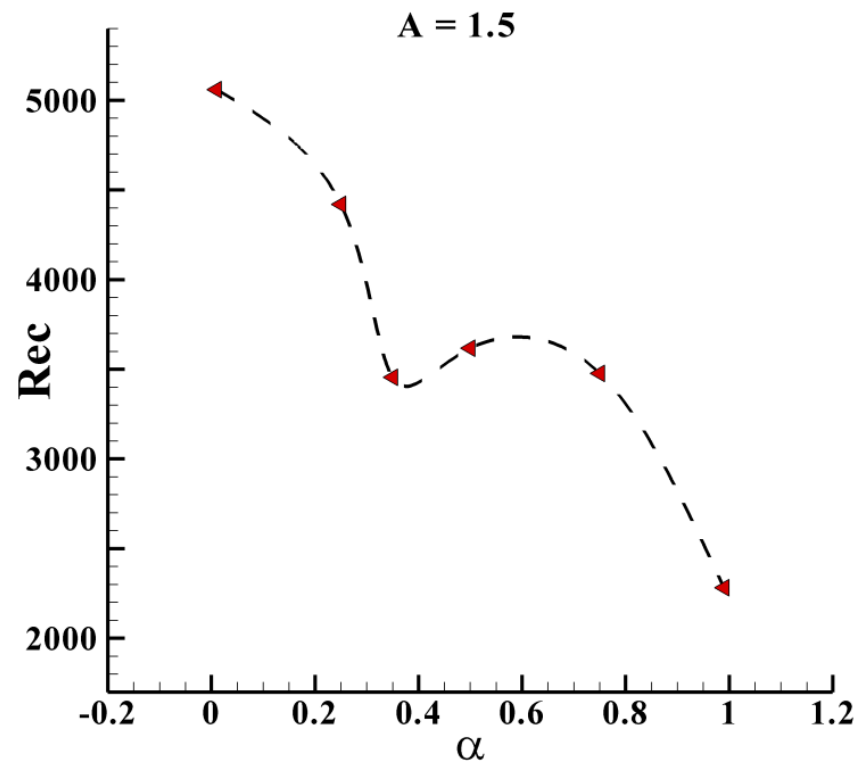

Fig. 10. Critical Reynolds number versus the velocity ratio $\alpha$, at $A=1.5$. 
(a)

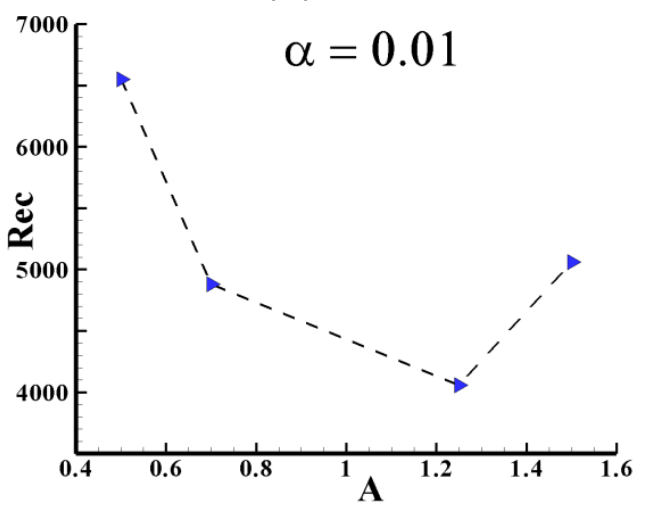

(c)

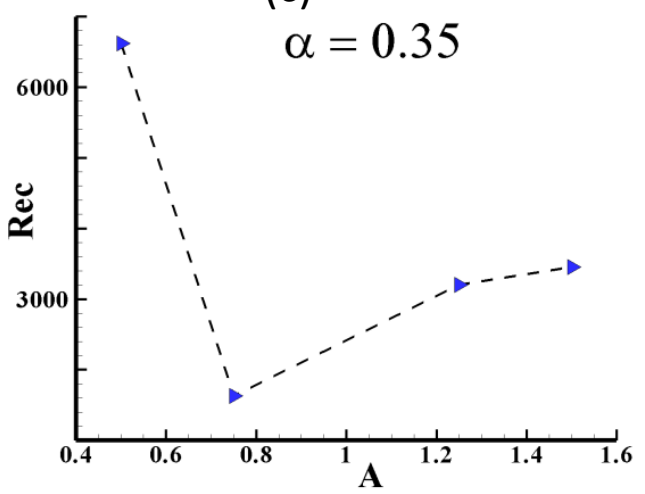

(e)

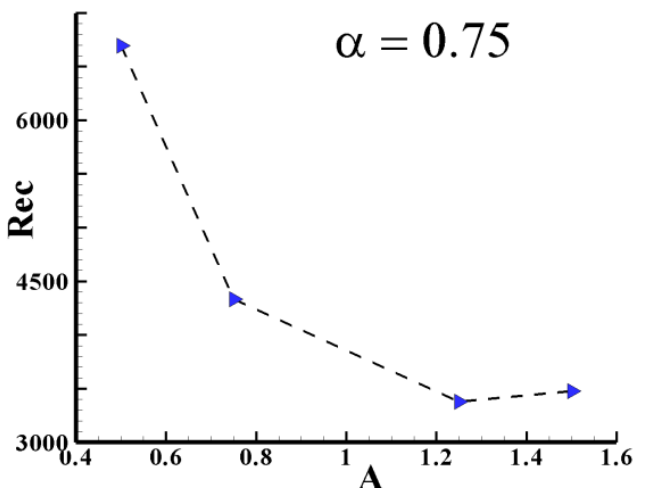

(b)

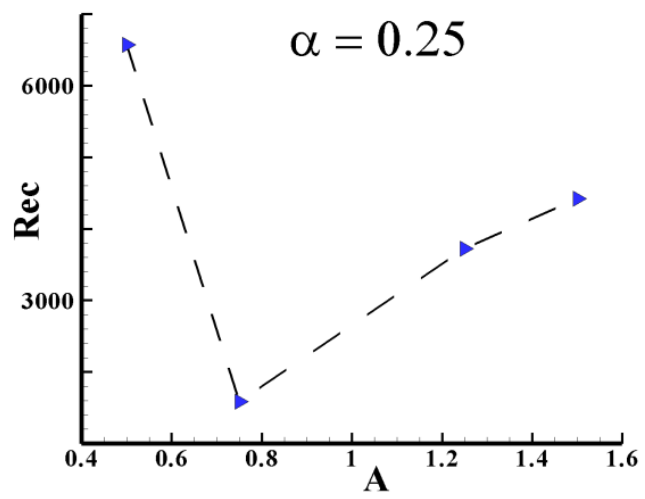

(d)

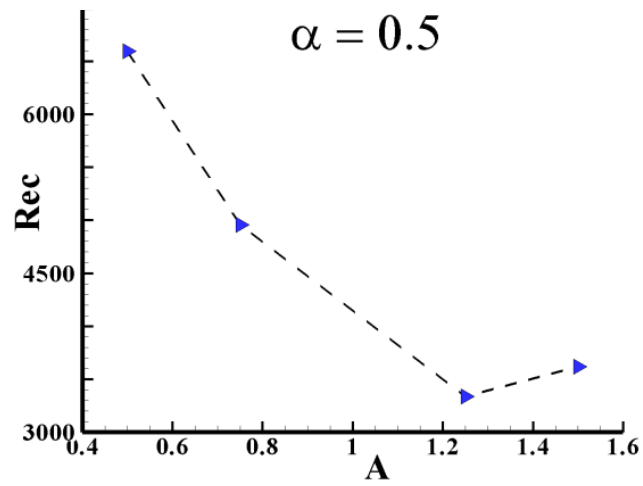

(f)

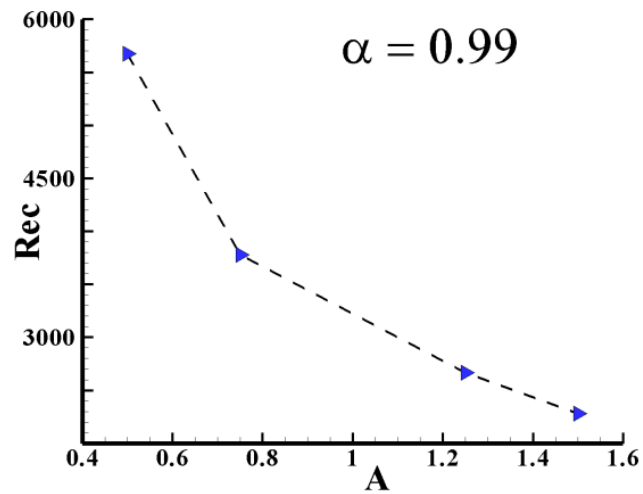

Fig. 11. Critical Reynolds number versus the aspect ratios A for the different corresponding values of $\alpha$.

Figure 11(a, b, c, d, e and f) shows the evolution of the critical Reynolds number as a function of the versus $A$ for different velocity ratios $\alpha$. As illustrated in this figure, Rec decreases and then increases by further augmenting the aspect ratios from $\mathrm{A}=0.25$ to $\mathrm{A}=1.5$. Similar variations are illustrated for the couple $(\alpha=0.25, \alpha=0.35)$ and $(\alpha=0.5, \alpha=0.75)$. At $\alpha=0.01$, the two sided driven cavity flow seems to approach the one sided one, while at $\alpha=0.99$ the cavity is approximated to a two-sided lid-driven cavity flow. Furthermore, the variation of the critical Reynolds number as a function of the aspect ratios exhibits different behavior for each velocity ratio $\alpha$. 


\section{CONCLUSION}

In this study, linear stability analysis of the lid-driven cavity flow has been performed for various Reynolds numbers. Two cases has been considered $(\alpha=1)$ for different aspect ratios $\mathrm{A}$ in the range $0.25 \leq \mathrm{A} \leq 1$ and four aspect ratios $\mathrm{A}(\mathrm{A}=0.5, \mathrm{~A}=0.75 \mathrm{~A}=1.25$, and $\mathrm{A}=1.5)$ with a large velocity ratio $\alpha$ examined in the range $0.01 \leq \alpha \leq 0.99$. In this paper, we have applied a QUICK type advection scheme and a finite volume method to explore in depth the vulnerability of the fluid flows to the impressed Reynolds number. Multigrid is adequately used as convergence accelerator to deliver numerical solutions on a non uniform staggered grid.

The bifurcations between steady and unsteady states are investigated. The effects of the three parameters $\{\mathrm{Re}, \mathrm{A}, \alpha\}$ on the bifurcates points has been analyzed. It is particularly found that Rec augments as $\alpha$ is increased from $\alpha=0.01$ to $\alpha=0.825$, then decreases to reach its minimum value $(\operatorname{Rec}=5673.08)$ at $\alpha=0.99$ with respect to the fixed $A=0.5$. Besides, Rec was seen to decrease as $\alpha$ increased from 0.01 to 0.35 and presents a minimum value $(\operatorname{Rec} \approx 1011.07)$ at $\alpha \approx 0.3$ according to $\mathrm{A}=0.75$. On another hand, similar plots of the variations of Rec versus the aspect ratio $\mathrm{A}$ were observed for the couple $(\alpha=0.25, \alpha=0.35)$ and $(\alpha=0.5, \alpha=0.75)$. Furthermore, at $\alpha=0.01$, the two sided driven cavity flow seems to approach the one sided one, while at $\alpha=0.99$ the cavity is approximated to a two-sided lid-driven cavity flow.

\section{References}

[1] Shankar PN, Deshpande MD. Fluid mechanics in the driven cavity. Annu Rev Fluid Mech 32 (2000) 93-136.

[2] Burggraf OR. Analytical and numerical studies of the structure of steady separated flows. J Fluid Mech 24 (1966) 113-51.

[3] Fortin A, Jardak M, Gervais JJ, Pierre R. Localization of Hopf bifurcations in fluid flow problems. Int. J. Numer. Methods Fluids; 24(11) (1997) 1185-1210.

[4] Erturk, E., Corke, T.C., and Gökcöl, C. Numerical solutions of 2-D steady incompressible driven cavity flow at high Reynolds numbers. International Journal for Numerical Methods in Fluids 48 (2005) 747-774.

[5] H. Ding, C. Shu, K.S. Yeo, D. Xu, Numerical computation of three-dimensional incompressible viscous flows in the primitive variable form by local multiquadric differential quadrature method, Comput. Methods Appl. Mech. Engrg. 195 (2006) 516-533.

[6] H.C. Kuhlmann, M. Wanschura, H.J. Rath, Flow in two-sided liddriven cavities: nonuniqueness, instabilities, and cellular structures, J. Fluid Mech. 336 (1998) 267-99.

[7] H.C. Kuhlmann, M. Wanschura, H.J. Rath, Elliptic instability in two-sided lid-driven cavity flow, Eur. J. Mech. B/Fluids 17 (1998) 561-69.

[8] Patil, D., Lakshmisha, K., Rogg, B., Lattice Boltzmann simulation of lid-driven flow in deep cavities, Comput Fluids 35(10) (2006) 1116 -1125.

[9] Taneda S. Visualization of separating Stokes flows. J. Phys. Soc. Jpn. 46 (1979) 1935-42.

[10] E.M. Wahba, Multiplicity of states for two-sided and four-sided lid driven cavity flows, Comput. \& Fluids 2008; 38:247-253.

[11] Arumuga Perumal, D., Dass, A.K.: Multiplicity of steady solutions in two-dimensional liddriven cavity flows by lattice Boltzmann Method. Comput. Math. Appl. 61 (2011) 3711-3721. 
[12] Perumal, D.A., Dass, A.K: Simulation of incompressible flows in two-sided lid-driven square cavities. Part I - FDM, CFD Letters, Issue 1, vol. 2 (2010) pp. 13-24. [13] A. Fortin, M. Jardak, J.J. Gervais, R. Pierre, Localization of Hopf bifurcations in fluid flow problems, Internat. J. Numer. Methods Fluids 24 (11) (1997) 1185-1210. [14] Y.F. Peng, Y.H. Shiau, R.R. Hwang, Transition in a 2-D lid-driven cavity flow, Comput. \& Fluids 32 (2003) 337-352.

[15] V.B.L. Bopanna, J.S.B. Gajjar, Global flow instability in a lid-driven cavity, Internat. J. Numer. Methods Fluids 62 (8) (2010) 827-853.

[16] S.V. Patankar, A calculation procedure for two-dimensional elliptic situations, Numer. Heat Transfer, 34 (1981) 409-425.

[17] Y. Achdou, J.L. Guermond, Convergence analysis of a finite element projection/Lagrange Galerkin method for the incompressible Navier-Stokes equations, SIAM J. Numer. Anal. 37 (2000) 799-826.

[18] B.P. Leonard, A stable and accurate convective modelling procedure based on quadratic upstream interpolation, Comput. Methods Appl. Mech. Eng., 19 (1979) 59. [19] R. Barrett, M. Berry, T.F. Chan, et al., Templates for the Solution of Linear Systems: Building Blocks for Iterative Methods, SIAM, 1994.

[20] N. Ben-Cheikh, B. Ben-Beya, T. Lili, Benchmark solution for time-dependent natural convection flows with an accelerated full-multigrid method, Num. Heat Trans. (B), 52 (2007) 131151.

[21] Reyad Omari, CFD simulation of Lid Driven cavity flow at moderate Reynolds Number, European Scientific Journal 9 (15) (2013) 45- 60. 